\title{
Ion Beam Notcher Using a Laser
}

\author{
R. Tomlin, FNAL, Batavia, IL60510, USA
}

\section{Abstract}

The FNAL LINAC will soon be asked to produce beam at $7.5 \mathrm{~Hz}$. FNAL LINAC extraction involves sweeping the $\mathrm{H}$-minus beam over a Lambertson magnet. The higher repetition rates are expected to activate the Lamberston magnet. A pulsed laser has been installed to make a notch in the beam so that beam will not sweep over the magnet.

\section{INTRODUCTION}

The first electron on $\mathrm{H}^{-}$has a binding energy of approximately $0.74 \mathrm{eV}$. We use an Nd:Yag laser having $1.165 \mathrm{eV}$ photons, $\lambda=1064 \mathrm{~nm}$, to ionize hydrogen ions that are streaming out of the $750 \mathrm{Kev}$ source at $0.04 \mathrm{c}$.

\section{GEOMETRY}

\subsection{General Layout}

The $H^{-}$ions are separated from photoionized $H^{0}$ by steering through a subsequent magnetic field. In this case we use a 90 degree dipole. Doglegs are ideal design tools for these reactions because the light can be made to collide or chase relativistic beam. That affords the option of wavelength shifting the laser to the particle frame, allowing a wider range of laser options.

\subsection{The Interaction Region}

Laser interactions with relativistic beams or beams that will be accelerated can be thought of as statistical chemical processes. Stripping is directly proportional to time that the ion beam remains in the light and the number of photons in the interaction region. For beam with velocity $0.04 \mathrm{c}$ the mixing time is essentially the laser pulse width. One can then run the laser pulse along a desired ion beam path by shooting across the ion beam or the ion beam can be "four-bumped" and the laser applied in colliding or following mode. The following mode allows the laser and the $H^{0}$ to share a common dump. The colliding mode was used in this application to take advantage of the existing beam line layout. The inside surface of an existing multiwire can serves the laser dump. $H^{0}$ land on the vacuum window that admits the laser pulse.

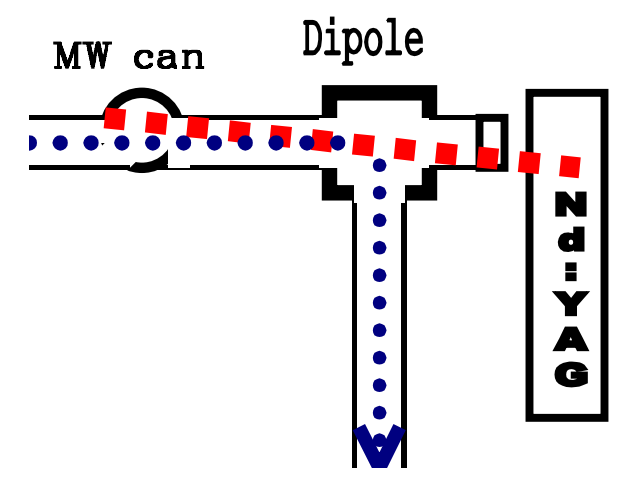

Figure 1

\subsection{The light}

A Surelite I-20 laser fires $400 \mathrm{~mJ}, 5$ nanosecond pulses at $15 \mathrm{~Hz}$. Pulse peak power is more than 80 megawatts and average power is 6 watts. It is mounted on a $2 \times 3$ foot optical table. The $6 \mathrm{~mm}$ diameter beam is directed with two 1 inch mirrors to a $4 \mathrm{X}$ expander on an $\mathrm{X}-\mathrm{Y}$ stage. The expanded beam is routed via 3 motorized mirrors through a coated vacuum window. These 2 and 3 inch mirrors can change the horizontal position, horizontal angle and vertical angle of the laser beam. The expanded beam is about $24 \mathrm{~mm}$ in diameter to cover the largest ion beam width found in the interaction region.

\subsection{Alignment}

Optics were assembled and tested in an interlocked laser lab. The range of angles vs. stepper motor counts was measured over a 4 foot path. The beam was set midrange in all three motor axis. The installation is in a public area and is completely shrouded. No laser beam can be used for spotting or alignment. The shrouded unit was then coupled to the vacuum window on the beam line and aligned with a ruler and dead reckoning. A $88 \%$ notch was seen on the beam on the first try.

\subsection{The optics}

Mirrors have $1064 \mathrm{~nm}$ coatings rated for $10 \mathrm{~J} / \mathrm{cm}^{2}$. The beam expander is rated at $8 \mathrm{~J} / \mathrm{cm}^{2}$. The laser has a $6 \mathrm{~mm}$ rod that produces diffraction rings of $30 \%$ depth in the near field. The net result is that we burned diffraction patterns in mirror coatings while testing at $450 \mathrm{~mJ}$ in 4.5 nsec pulses at $20 \mathrm{~Hz}$. The fix was to reduce power, go 15 $\mathrm{Hz}$, and rotate a good spot on the mirror into the beam. 
The vacuum window is one inch BK-7 glass AR coated for $1064 \mathrm{~nm}$ and mounted with O-rings in a 3 inch I.D. vacuum flang.

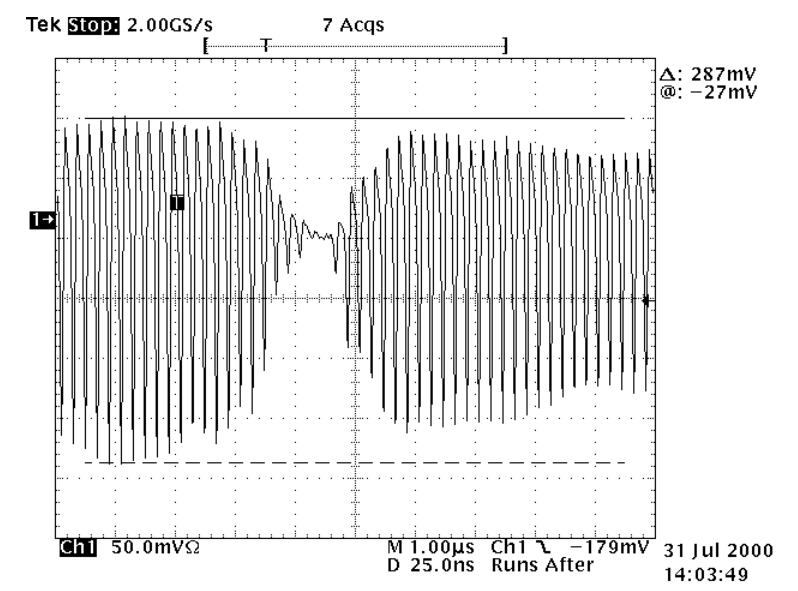

Figure 2: $200 \mathrm{MHz}$ bunch signal from a BPM plate at the end of RF tank two in FNAL Linac shows a $99 \%$ notch. This is a $200 \mathrm{~mJ}, 5 \mathrm{~ns}$ pulse crossing at an angle.

\section{WAVE LENGTH AND POWER CALCULATIONS}

\subsection{Laser wavelength calculation}

Broad \& Reinhardt calculated the photoionization cross section of H- ions. [1] Their work shows a broad photoelectric peak for $1.5 \mathrm{eV}$ photons with a reaction cross section or area of $4 \times 10^{-17} \mathrm{~cm}^{2}$ for each ion. This is roughly half the area which can be calculated from the Bohr radius.

Then $0.7545 \mathrm{eV} \times 1.602 \times 10^{-19} \mathrm{~J} / \mathrm{eV}=1.2 \times 10^{-19} \mathrm{~J}$

That is the quantum energy required by a photon to kick the electron loose in the reaction $H^{-}+\lambda \rightarrow H_{0}+e$. Note that the binding energy is $0.75451 \mathrm{eV}$ but the reaction becomes more efficient nearer $1.5 \mathrm{eV}$. The "rest frame" wave length is hc/E:

$\lambda=\frac{6.626 \times 10^{-34} \mathrm{~J} \cdot \mathrm{s} \times 2.998 \times 10^{8} \mathrm{~m} / \mathrm{s}}{1.2 \times 10^{-19} \mathrm{~J}}$

$\lambda=1643 \mathrm{~nm}$ for resting $\mathrm{H}$ - ions. $750 \mathrm{KeV}$ ions travel at $0.04 \mathrm{c}$. The wavelength required in the lab or laser frame for colliding beams is:

$\lambda_{\text {laser }}=\lambda_{\text {ion }} \cdot \gamma(1-\beta \cos \theta)$

where $\cos \theta=1$

$\beta=v / c, \quad \gamma=1 / \sqrt{1-\beta^{2}}$

$\lambda_{\text {laser }}=1643 \mathrm{~nm} \cdot(1.0008)(1-0.04)$

$\lambda_{\text {laser }}=1579 \mathrm{~nm}$

A $1064 \mathrm{~nm}$ laser will work.

\subsection{Laser power calculation}

Power calculation is a statistical game. Making something of the analogy to dice on a table, let $\sigma=4 \times 10^{-17} \mathrm{~cm}^{2}$, the photo ionization cross section of an $\mathrm{H}$ - ion. If the die were n-sided, each side with area $\sigma$, and the area of the table top was $\mathrm{A}=\mathrm{n} \sigma$ (analogous to the cross section of the interaction region), the probability of the one photon landing on the one ion is: $P=\frac{\sigma}{A}, \quad N_{\gamma}=1, \quad H=1$

Lots of photons increases the odds of ionizing one $\mathrm{H}^{-}$;

$$
P=\frac{\sigma N_{\gamma}}{A}
$$

Lots of $\mathrm{H}^{-}$ions increased the odds of interaction even more;

$$
P=\frac{\sigma H N_{\gamma}}{A}
$$

The longer one throws the dice, the more throws one gets and the better the odds;

$P=\frac{\sigma H N_{\gamma}}{A} d t$

Meanwhile the laser is delivering photons per second evenly over ion beam cross section A;

$$
P=\sigma H \frac{N_{\gamma}}{A d t} d t
$$

But laser power is defined as

$$
I=N_{\gamma} \cdot \mathrm{cm}^{-2} \cdot \mathrm{s}^{-1}
$$

So: $\quad P=\sigma H I d t \quad$ is the probability of stripping.

The number of $H^{-}$remaining at any given time is proportional to the probability of stripping;

$d H=-\sigma H_{\text {init }} I d t \quad$ rearranging :

$\frac{d H}{H_{\text {init }}}=-\sigma I d t$

To get from point a to point $\mathbf{b}$ :

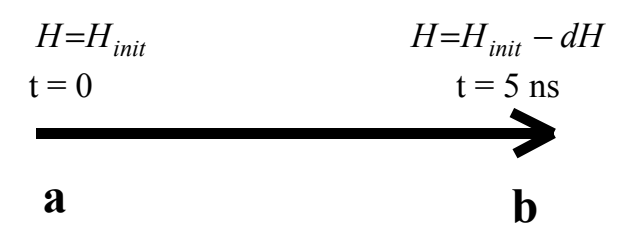

with $\mathrm{H}=H^{-}$left at any time t, integrate ...

$\int_{a}^{b} \frac{1}{H_{\text {init }}} d H=\int_{a}^{b}-\sigma I d t$

$\ln H=-\sigma I \quad t+C$

$H=e^{-\sigma I t} \cdot e^{c} \quad$ ions remaining

$H_{\text {init }}$ is about the only constant around, so: 
$\frac{H}{H_{\text {init }}}=e^{-\sigma I t}, \quad \ln \left(\frac{H}{H_{\text {init }}}\right)=-\sigma I t$

$\mathrm{H}$ is ions remaining. For $90 \%$ stripping $H / H_{\text {init }}=0.10$.

Nothing left to do but solve for photons per $\mathrm{cm}^{2}$ second needed to strip $90 \%$ of the ion beam during a 5 nanosecond laser pulse.

$I=\frac{\ln (0.10)}{-\sigma t}=\frac{-2.303}{-4 \times 10^{-17} \mathrm{~cm}^{2} \cdot 5 \times 10^{-9} \mathrm{sec}}$

$I=1.15 \times 10^{25} \frac{\text { photons }}{c m^{2} \text { second }}$

To maintain that flux over an area covering a $2 \mathrm{~cm}$ circle:

Area $=\pi \mathrm{cm}^{2}, \quad I=\pi I_{\text {init }}$

$I=3.62 \times 10^{25}$ photons $/ \mathrm{cm}^{2} \mathrm{sec}$

are required to strip $90 \%$ of an ion beam with cross section pi cm squared.

At $1 \mu m$ each photon has energy $\mathrm{E}$ :

$$
\begin{aligned}
& \mathrm{E}=\frac{\mathrm{h} \mathrm{c}}{\lambda}=\frac{6.626 \times 10^{-34} \mathrm{~J} \mathrm{~s} \cdot 2.998 \times 10^{8} \mathrm{~m} / \mathrm{s}}{1 \times 10^{-6} \mathrm{~m}} \\
& \mathrm{E}=1.88 \times 10^{-19} \mathrm{Joule} / \text { photon } \\
& \text { Joule } / \mathrm{sec} \quad=\text { Watt } \\
& \text { Watts }=1.88 \times 10^{-19} \frac{\text { Joule }}{\text { photon }} \\
& 3.62 \times 10^{25} \frac{\text { photons }}{\text { second }}
\end{aligned}
$$

Peak Power $=6.8 \mathrm{MW}$

Energy per pulse $=6795998 \mathrm{~J} / \mathrm{s} \times 5 \times 10^{-9} \mathrm{~s}$ $=34 \mathrm{~mJ}$

That means that the laser must deliver $6.8 \mathrm{MW}$ at $1 \mu \mathrm{m}$ for the $5 \mathrm{nsec}$ that a laser pulse bathes the interaction region to get $90 \%$ stripping.

There are so many more photons than ions that photon depletion is not a problem. This photon flux will strip $90 \%$ of any intensity ion beam obtainable. The intensity profiles of the colliding laser and ion beams are assumed to be similar.

Care may be taken to define the interaction region and pulse length so that ions don't see photons in magnetic fields.

Multiple reflection cavities tend to be unstable. Very careful consideration must be given to system geometry, keeping in mind that to get more stripping, the ions must remain in the photon brew longer or the photon brew must be stronger.

Laser pulses can be reused if stored until needed. We have proposed a storage time of 2.22 usec in a four pass bow-tie cavity some 665 meters long. Large Pockel's cells would shuttle the pulse to and from the delay line. A disk gain section would restore optical losses. The $15 \mathrm{~Hz}$ YAG could then drive our desired bursts of 12 pulses at $15 \mathrm{~Hz}$.

Power levels required vs. \% ions stripped per $\pi \mathrm{cm}^{2}$ are given in the following table:

Table 1: Laser power vs. stripping

\begin{tabular}{|c|c|c|}
\hline $\begin{array}{c}\text { Peak } \\
\text { Watts }\end{array}$ & $\begin{array}{c}\text { \% } \\
\text { Stripped }\end{array}$ & $\begin{array}{c}\text { millijoules } \\
\text { Average Energy }\end{array}$ \\
\hline 671182 & 20 & 3 \\
\hline 2084883 & 50 & 10 \\
\hline 13851665 & 99 & 69 \\
\hline 20777498 & 99.9 & 103 \\
\hline
\end{tabular}

\section{REFERENCES}

[1] John T. Broad \& William P. Reinhardt, "One and two electron photoejection from H-: a multichannel Jmatrix calculation" Phys. Rev. A, Vol 14, N0. 6, Dec 1976 , page 2159 .

[2] Ray Tomlin, "Laser Stripping of Relativistic $H^{-}$ Ions With Practical Considerations” FNAL TM1957. 